\title{
A focus on direct oral anticoagulants: "old" and possible new indications and efforts for a better clinical management
}

\author{
Domenico Prisco ${ }^{1,2}$
}

Received: 3 August 2018 / Accepted: 18 September 2018 / Published online: 21 September 2018

(c) Società Italiana di Medicina Interna 2018

Keywords Direct oral anticoagulants · Atrial fibrillation · Venous thromboembolism · Renal function · Bleeding · Intracranial hemorrhage

Both platelets and coagulation play key roles in initiation, amplification, and perpetuation of acute coronary syndromes (ACS). Nonetheless, patients with ACS frequently have recurrent ischemic events despite the use of currently recommended dual antiplatelet therapy, revascularization procedures as appropriate, and other evidence-based secondary preventive measures. Numerous antithrombotic cocktails including oral anticoagulants (OACs) with or without aspirin have been tested in large clinical trials with the goal of further reduction of ischemia and bleeding risk.

In the paper entitled "Clinical effects with inhibition of multiple coagulative pathways in patients admitted for acute coronary syndrome" [1], Ilaria Cavallari and Giuseppe Patti review clinical outcomes resulting from inhibition of multiple coagulative pathways in patients with ACS in the light of data from the literature. They comment that the optimal balance between the reduction of ischemic events and the induction of bleeding remains a major challenge. Future clinical trials need to address remaining important clinical issues, such as early switching from P2Y12 inhibitors to low-dose anticoagulation on top of aspirin, more potent P2Y12 inhibitors in the context of triple therapy and lowdose ticagrelor in ACS patients.

Over the past few years, non-vitamin $\mathrm{K}$ antagonist oral anticoagulants (NOACs), also called direct oral anticoagulants (DOACs), targeting directly a single activated clotting factor, have been developed for the prophylaxis of cardioembolic stroke in patients with non-valvular atrial fibrillation

Domenico Prisco

priscod@aou-careggi.toscana.it

1 Department of Experimental and Clinical Medicine, University of Florence, Florence, Italy

2 Interdisciplinary Internal Medicine, Careggi University Hospital, Florence, Italy
(AF), and are currently recommended as first-line therapy in AF. In the article entitled "NOAC in real world patients with atrial fibrillation in Italy: results from the ISPAF-2 (Indagine Sicoa Paziente con Fibrillazione Atriale) survey study" [2], Maurizio Volterrani and colleagues try to provide an overall picture on the extent to which NOACs are prescribed in an unselected population of consecutive patients with AF in Italy, also evaluating the compliance with therapy and the quality of life. Data reported come from a cardiology network enrolling a total of 1742 consecutive AF outpatients with a mean age of 72.5 years. The ISPAF-2 study shows that the prescription of OAC according to current guidelines and stroke-risk scoring system is rather high in the real world, although it still needs to be improved. As already observed in other surveys, in a high proportion of low-risk patients, according to CHA2DS2VASc, anticoagulation therapy, with either NOACs or vitamin $\mathrm{K}$ antagonists (VKAs), is still prescribed, making it necessary to have educational programs for appropriate treatments.

In the accompanying editorial commentary "The changing landscape of thromboprophylaxis for atrial fibrillation: insights from the ISPAF-2 survey" [3], Giuseppe Mule' and colleagues report that some data of the survey are in contrast with those of other studies, but they comment that inconsistent results suggest important methodological differences among these studies. They also highlight the inherent problems with interpreting data from registries that may not be entirely representative of the general population.

Prescription of NOACs requires an assessment of renal function (RF) and the Cockcroft-Gault (CG) equation is traditionally recommended. The objective of the observational study reported in the article of Vincenzo Livio Malavasi and colleagues entitled "Variations in clinical management of non-vitamin $\mathrm{K}$ antagonist oral anticoagulants in patients with atrial fibrillation according to different equations for 
estimating renal function" [4] is to evaluate the potential changes in NOAC management using five different equations for estimating RF. Authors find that the adoption of the equations proposed for estimating glomerular filtration rate (GFR) instead of the CG equation implies that in every 6-9 patients, the different classification of RF results in a substantially different management of NOACs in terms of drug dosing or even avoidance of prescription according to evidence-based recommendations. Moreover, the most pronounced changes in RF classification are detected in patients $\geq 75$ years. In view of the vulnerability and frailty of most of these patients, further studies on NOAC dosing according to the equations available for GFR evaluation should be promoted in this setting, as well as at the extremes of BMI. Interesting comments on this topic can be found in the Editorial commentary by Riva, Ageno and Gatt entitled "Estimating renal function in patients with atrial fibrillation: which dose of direct oral anticoagulants?" [5]

After the publication of an Italian Intersociety Consensus on the use of DOACs in our Journal [6], Doris Barcellona and Francesco Marongiu are the authors of a "point-of-view article" entitled "Direct oral anticoagulants: what can we learn?" [7] in which they comment some of the topics covered by the Consensus. First of all, authors underline that a careful examination of the performance of DOACs versus warfarin in randomized clinical trials dedicated to AF reveals a poor performance of warfarin because the percentage of major bleeding is always above $3 \%$; however, the percentage of major bleeding is less than half of that when the management is done in anticoagulation clinics (ACs). Authors discuss the transformation of ACs into thrombosis centers (TCs) to cover many new activities in terms of diagnostic tools and therapeutic choices. Their role may be crucial in selecting both the most appropriate diagnostic approach and the best therapeutic option for the single patient. TCs can also organize a regular follow-up to improve patient adherence to DOACs. According to the authors, marketing might have a role in the decision-making of the individual doctor so that efforts should be made for limiting the relationships between doctors and pharmaceutical companies.

Prognosis after mild traumatic brain injury (MTBI) on OACs is uncertain. In the article entitled "Intracranial hemorrhage in anticoagulated patients with mild traumatic brain injury: significant differences between direct oral anticoagulants and vitamin K antagonists", Alessandro Cipriano and colleagues [8] evaluate the rate of immediate and delayed traumatic intracranial hemorrhage ( $\mathrm{ICH})$ and the safety of a clinical management protocol in a single-center prospective observational study, enrolling 220 patients on OACs with MTBI. As expected, DOACs are safer than VKAs also in the setting of MTBI. In this study, the rate of delayed hemorrhage is relatively low. According to these results, patients presenting with a negative initial CT scan and without neurological deterioration could be safely discharged after a short period of in-ward observation with a low rate of complications and without a second CT scan.

Despite the availability of updated guidelines for the diagnosis and treatment of venous thromboembolism (VTE), the management of this disorder in clinical practice is often not standardized. In the article entitled "Multidisciplinary approach to the management of patients with pulmonary embolism and deep vein thrombosis: a consensus on diagnosis, traditional therapy and therapy with rivaroxaban" [9], Davide Imberti and a board of experts in VTE try to define strategies to improve the quality of patient care and the efficiency of healthcare resource utilization. The proposal of shared strategies between emergency departments (ED), different specialists and general practitioners (GP) for the correct management of patients with suspected VTE would have the triple objective of (a) ensuring a rapid confirmation of the diagnosis and preparation of a proper treatment plan; (b) reducing inappropriate hospitalizations, and (c) rationalizing the processes and timing for outpatients' management. In the perspective to implement these algorithms, as well as to optimize the multidisciplinary management of VTE patients, authors advise the adoption of an integrated file (like the one proposed by the King's College Hospital, NHS Foundation Trust) to follow these patients from their first doctor's visit (GPs, ED, etc.), allowing for progressive and constant monitoring of the undertaken interventions by the various specialists involved in VTE management. Among NOACs, in this paper authors focused on rivaroxaban only because it was the unique available NOAC for the treatment of VTE in Italy at the time the manuscript was written, which is no longer the case.

Another relevant topic for DOACs is their possible role in VTE prophylaxis in acutely ill medical patients. The topic is controversial and is the object of a debate.

The "pro" reasons are reported by Walter Ageno in the article entitled "Has time come for the use of direct oral anticoagulants in the extended prophylaxis of venous thromboembolismin acutely ill medical patients? Yes" [10]. He reports the positive results of APEX trial performed with betrixaban [11] underlining that the analysis of the overall study population shows a favorable net clinical benefit with betrixaban, with a statistically significant reduction in all efficacy outcomes and no increase in major bleeding rates. The reasons against are given by Francesco Marongiu and Doris Barcellona in the article entitled "Has time come for the use of direct oral anticoagulants in the extended prophylaxis of venous thromboembolism in acutely ill medical patients? No" [12]. According to the authors, betrixaban seems to be a very promising oral anticoagulant dedicated to critical patients, since the risk of bleeding appears generally low. However, they think that other phase III studies and post-marketing registers are required before accepting 
betrixaban among the different anticoagulant options in daily practice. An interesting editorial commentary on this debate entitled "Has time come for the use of direct oral anticoagulants in the extended prophylaxis of venous thromboembolism in acutely ill medical patients?" [13] has been written by Franco Piovella and Diana Irina Iosub who focus in particular on the role of D-dimer and discuss the different behavior of Food and Drug Administration, and European Medicines Agency in the approval of betrixaban for this indication.

Elisa Maria Fiorelli and Roberta Elisa Rossi on behalf of GrAM (Gruppo di Autoformazione Metodologica) in the "Cutting edge: research update" article entitled "Edoxaban for the treatment of cancer associated venous thromboembolism as an alternative to low molecular weight heparin" [14] analyze the results of the Hokusai cancer trial [15] and discuss some points of strength and weakness of this important study.

The management of major bleeding in patients treated with DOACs is still not well established. START-SSC Events, a branch of the START registry (Survey on anTicoagulated pAtients RegisTer) [16] promoted through the Scientific and Standardization Committee (SSC) Control of Anticoagulation of the International Society of Thrombosis and Haemostasis, aims to describe the actual management of bleeding or recurrent thrombotic events in patients treated with the DOACs in routine clinical practice. In the article entitled "Management of major bleeding and outcomes in patients treated with direct oral anticoagulants: results from the START-Event registry" [17], Sophie Testa and colleagues describe the management of major bleeding with respect to therapeutic strategies (medical therapies, surgery, or interventional procedures), laboratory testing, and other supportive measures, and the adherence to currently available guidelines and recommendations in clinical practice. Between January 2015 and December 2016, 117 patients with major bleeding events were enrolled. AF was the indication for treatment in most of them; 53 patients had intracranial bleeding (13 fatal), 42 had gastrointestinal bleeding ( 1 fatal), and 22 had bleeding in other sites. Therapeutic interventions for the management of bleeding were performed in $71 \%$ of patients. The authors report a high heterogeneity in the management of bleeding complications in patients treated with DOACs, and conclude by suggesting some unmet needs in the field: [1] homogeneous and more structured guidelines; [2] availability of reversal agents; [3] DOAC-specific blood measurements, rapidly available in emergency; [4] specific training on anticoagulation reversal for emergency physicians; and [5] available consultant expert on thrombosis and haemostasis, who may ensure upgraded, homogeneous, and probably more effective management of acute major bleeding complications in anticoagulated patients.
DOACs possess high bioavailability, and their anticoagulant effect is more predictable than that of VKAs, hence they do not require routine dose adjustment based on laboratory testing. However, there are circumstances when laboratory testing may be useful, including patients who need to undergo surgery or invasive procedures. This topic is treated in the review entitled "The vexed question of whether or not to measure levels of direct oral anticoagulants before surgery or invasive procedures" by Armando Tripodi and colleagues [18], who discuss the pros and cons of measuring (or not measuring) DOAC levels before surgery or invasive procedures. The authors are a multidisciplinary team of experts with different backgrounds, including the thrombosis laboratory, clinical thrombosis, internal medicine, cardiology and nephrology. The conclusion is that measuring DOACs with dedicated tests before surgical or invasive procedures is important for patient safety because it provides the best and most direct evidence to rule in (or to rule out) clinically relevant concentrations of residual drugs. According to authors' suggestions, regulatory agencies should urgently approve their use in clinical practice, hospital administrators should make them available, and clinical laboratories should setup the relative methods and make them available to clinicians.

In the Editorial commentary "The vexing conundrum: to measure direct oral anticoagulants before surgery or invasive procedures or not" [19], Jim Douketis and Mansoor Radwi make interesting considerations. They say that the over-arching issue is whether a strategy of measuring DOAC levels confers a net clinical benefit above that of a no measurement strategy and that a plausible hypothesis is that a DOAC measurement-based strategy would benefit over a no measurement strategy. According to authors, one could design a randomized trial to compare the two strategies, but what is currently lacking, are reliable estimates of event rates with a no measurement strategy approach that would form the control group in such a trial.

In the letter entitled "Drug plasma level measurement in management of severe bleeding during direct oral anticoagulant treatment: case report and perspective" [20], Dimitriy Arioli and colleagues propose an algorithm to treat bleeding in patients on DOACs. Authors conclude that readily available DOAC plasma level measurement may become a useful tool to make rational decisions in emergency contexts. Protocols should include it for the management of patients with severe bleeding, at least in those centers where severe complications of patients receiving antithrombotic therapy are likely to be managed.

I hope that this focus will be of interest for the readers. 


\section{Compliance with ethical standards}

Conflict of interest The author declares that he has no conflict of interest.

Statements on human and animal rights This article does not contain any studies with human participants or animals.

Informed consent None.

\section{References}

1. Cavallari I, Patti G (2018) Clinical effects with inhibition of multiple coagulative pathways in patients admitted for acute coronary syndrome. Intern Emerg Med. https://doi.org/10.1007/s1173 9-018-1834-x

2. Volterrani M, Iellamo F, Cappelletti A, Alcamo P, Pezzullo S, Piccinini M, Miano M, Urso L, Proto C, Ricciardelli B, Pusineri E, on behalf of SICOA (Società Italiana Cardiologia Ospedalità Accreditata) Investigators (2018) NOAC in "real world" patients with atrial fibrillation in Italy: results from the ISPAF-2 (Indagine Sicoa Paziente Con Fibrillazione Atriale) survey study. Intern Emerg Med. https://doi.org/10.1007/s11739-018-1896-9

3. Mulè G, Carollo C, Guarneri M, Cottone S (2018) The changing landscape of thromboprophylaxis for atrial fibrillation: insights from the ISPAF-2 survey. Intern Emerg Med. https://doi. org/10.1007/s11739-018-1929-4

4. Malavasi VL, Pettorelli D, Fantecchi E, Zoccali C, Laronga C, Trenti T, Lip GYH, Boriani G (2018) Variations in clinical management of non-vitamin $\mathrm{K}$ antagonist oral anticoagulants in patients with atrial fibrillation according to different equations for estimating renal function. Intern Emerg Med. https://doi. org/10.1007/s11739-018-1857-3

5. Riva N, Ageno W, Gatt A (2018) Estimating renal function in patients with atrial fibrillation: which dose of direct oral anticoagulants? Intern Emerg Med. https://doi.org/10.1007/s1173 9-018-1883-1

6. Prisco D, Ageno W, Becattini C, D'Angelo A, Davì G, De Cristofaro R, SIMI (Italian Society of Internal Medicine), FADOI (Federation of Associations of Hospital Doctors on Internal Medicine), SISET (Italian Society for the Study of Haemostasis and Thrombosis) et al (2017) Italian intersociety consensus on DOAC use in internal medicine. Intern Emerg Med 12(3):387-406

7. Barcellona D, Marongiu F (2018) Direct oral anticoagulants: what can we learn. Intern Emerg Med. https://doi.org/10.1007/s1173 9-018-1811-4

8. Cipriano A, Pecori A, Bionda AE, Bardini M, Frassi F, Leoli F, Lami V, Ghiadoni L, Santini M (2018) Intracranial hemorrhage in anticoagulated patients with mild traumatic brain injury: significant differences between direct oral anticoagulants and vitamin K antagonists. Intern Emerg Med. https://doi.org/10.1007/s1173 9-018-1806-1
9. Imberti D, Becattini C, Bernardi E, Camporese G, Cuccia C, Dentali F, Paretti D (2018) Multidisciplinary approach to the management of patients with pulmonary embolism and deep vein thrombosis: a consensus on diagnosis, traditional therapy and therapy with rivaroxaban. Intern Emerg Med. https://doi.org/10.1007/ s11739-018-1802-5

10. Ageno W (2018) Has time come for the use of direct oral anticoagulants in the extended prophylaxis of venous thromboembolismin acutely ill medical patients? Yes. Intern Emerg Med. https:// doi.org/10.1007/s11739-017-1723-8

11. Cohen AT, Harrington RA, Goldhaber SZ et al (2016) Extended thromboprophylaxis with betrixaban in acutely ill medical patients. N Engl J Med 375:534-544

12. Marongiu F, Barcellona D (2018) Has time come for the use of direct oral anticoagulants in the extended prophylaxis of venous thromboembolism in acutely ill medical patients? No. Intern Emerg Med. https://doi.org/10.1007/s11739-018-1843-9

13. Piovella F, Iosub DI (2018) Has time come for the use of direct oral anticoagulants in the extended prophylaxis of venous thromboembolism in acutely ill medical patients? Intern Emerg Med. https://doi.org/10.1007/s11739-018-1953-4

14. Fiorelli ME, Rossi RE, GrAM (Gruppo di Autoformazione Metodologica) (2018) Edoxaban for the treatment of cancer associated venous thromboembolism as an alternative to low molecular weight heparin. Intern Emerg Med. https://doi.org/10.1007/ s11739-018-1886-y

15. Raskob GE, van Es N, Verhamme $P$ et al (2018) Edoxaban for the treatment of cancer-associated venous thromboembolism. N Engl J Med 378:615-624

16. Antonucci E, Poli D, Tosetto A et al (2015) The Italian STARTregister on anticoagulation with focus on atrial fibrillation. PLoS One 10:e124719

17. Testa S, Ageno W, Antonucci E, Morandini R, Beyer-Westendorf J, Paciaroni M, Righini M, Sivera P, Verhamme P, Pengo V, Poli D, Palareti G (2018) Management of major bleeding and outcomes in patients treated with direct oral anticoagulants: results from the START-Event registry. Intern Emerg Med. https://doi. org/10.1007/s11739-018-1877-Z

18. Tripodi A, Marongiu F, Moia M, Palareti G, Pengo V, Poli D, Prisco D, Testa S, Zanazzi M (2018) The vexed question of whether or not to measure levels of direct oral anticoagulants before surgery or invasive procedures. Intern Emerg Med. https ://doi.org/10.1007/s11739-018-1854-6

19. Douketis JD, Radwi M (2018) A clinical conundrum: to measure or not measure direct oral anticoagulants before a surgery or procedure? Intern Emerg Med. https://doi.org/10.1007/s1173 9-018-1930-y

20. Arioli D, Donelli D, Morini L, Leone MC, Negri EA (2018) Drug plasma level measurement in management of severe bleeding during direct oral anticoagulant treatment: case report and perspective. Intern Emerg Med. https://doi.org/10.1007/s1173 9-018-1858-2 